\title{
Endoscopic management of Zenker's diverticulum: stapling vs. Z-POEM
}

\author{
Raffaele Cerchione, Paolo Parise, Greta Olivari, Andrea Cossu, Lavinia Barbieri, Marco Palucci, Lorenzo \\ Cinelli, Francesco Puccetti, Riccardo Rosati
}

Division of Gastrointestinal Surgery, IRCCS San Raffaele Scientific Institute, Milan, Italy

Contributions: (I) Conception and design: R Cerchione, P Parise, R Rosati; (II) Administrative support: R Cerchione, P Parise, R Rosati; (III) Provision of study materials or patients: R Cerchione, G Olivari, A Cossu, F Puccetti, L Barbieri, L Cinelli, M Palucci; (IV) Collection and assembly of data: R Cerchione, G Olivari, A Cossu, F Puccetti, L Barbieri, L Cinelli, M Palucci; (V) Data analysis and interpretation: R Cerchione, P Paolo, R Riccardo, A Cossu; (VI) Manuscript writing: All authors; (VII) Final approval of manuscript: All authors.

Correspondence to: Raffaele Cerchione. Division of Gastrointestinal Surgery, IRCCS San Raffaele Scientific Institute, Via Olgettina 60, Milan 20132, Italy. Email: cerchione.raffaele@hsr.it.

\begin{abstract}
Zenker's diverticulum (ZD) is a rare disease of the elderly. Surgery was the first treatment option for many years. Subsequently many different endoscopic treatments have been introduced in order to reduce the complication rate. Endoscopic stapled diverticulostomy (ESD) is a well-known procedure since 1993. Recently an endoscopic myotomy of the upper esophageal sphincter (UES), called per-oral-endoscopicmyotomy for Zenker's diverticulum (Z-POEM) and derived from the POEM technique for achalasia, has been proposed. The aim of this study is to describe and compare these two different techniques for treatment of ZD in order to assess their efficacy and safety. We reviewed literature of the last 10 years on 1,490 patients that underwent ESD from 11 different papers and on 96 patients from 9 papers that underwent Z-POEM. Surgical procedure techniques, clinical and technical success, recurrence rate and complications were reviewed. The most common complications are bleeding and perforations that most of time are selfresolving but sometimes could require a surgical intervention. Other minor but frequent complications are dental avulsions and lips tears. Only one death was described among those submitted to ESD. Both procedures are effective in symptoms solutions with a low recurrence rate. Anyway, the main drawback of ESD is the difficult positioning of the diverticuloscope, that is the main reason for technical failure. On the opposite data about Z-POEM are few and of low quality, so some concerns remain about symptoms such regurgitation and aspiration. Minimally invasive treatment of ZD with both ESD and Z-POEM is a valuable option for this disorder. They are both safe, feasible and effective, in terms of hospital stay, complication rate and clinical success rate. Long-term results have to be evaluated considering a larger population for the Z-POEM procedure.
\end{abstract}

Keywords: Zenker's diverticulum (ZD); stapling diverticulostomy; endoscopic stapling diverticulostomy; per-oralendoscopic-myotomy for Zenker's diverticulum (Z-POEM); flexible endoscopy

Received: 09 November 2019; Accepted: 17 January 2020; Published: 10 October 2020.

doi: $10.21037 /$ shc.2020.01.04

View this article at: http://dx.doi.org/10.21037/shc.2020.01.04

\section{Background}

Zenker's diverticulum (ZD) is a protrusion of the pharyngeal mucosa and submucosa through Killian's triangle, between the inferior pharyngeal constrictor muscle and the cricopharyngeal muscle. The underling mechanism of formation is the pulsion against a dyscinetic upper esophageal sphincter (UES). It is a rare disorder and mostly affects elderly people, but still, it is the most frequent esophageal diverticulum. Its incidence is reported to be 
2 per 100,000 per year (1).

The main symptoms are dysphagia and regurgitation that can lead to malnutrition and weight loss. Important is also the risk of aspiration pneumonia. History, symptoms and signs may suggest the presence of a ZD but only morphological studies such as $\mathrm{X}$-rays barium swallow and upper-G.I. endoscopy can prove its existence. Sometimes diagnosis occurs accidentally, while performing one of the previous tests for other reasons.

The mainstay of whatever treatment is the section of the muscular fibres of the UES. Standard surgical procedure is the myotomy of the cricopharyngeal muscle combined with different possible treatment of the diverticulum through a left neck incision. The first to perform a successful surgical treatment was Wheeler in 1886 (2). Since then, different open surgical procedures were described such as diverticulectomy, diverticulopexy and diverticular inversion. The first endoscopic treatment was done in 1917 by Mosher, who performed a septotomy with scissors; but a high mortality, due to mediastinitis, was reported (3). In 1993 Collard performed for the first time an esophagodiverticulostomy with a linear stapler through a rigid endoscope endoscopic stapled diverticulostomy (ESD) (4). The advantage of this technique was the "seal and cut" effect of the stapler, avoiding the risk of leakage. In 2016, Quan-Lin Li described for the first time the per-oralendoscopic-myotomy for Zenker's diverticulum (Z-POEM), a technique derived from the P.O.E.M. for achalasia, in which a partial septotomy is performed through a section of the muscular fibres of the UES (5). Aim of this study was to review the literature in order to compare ESD and Z-POEM in terms of safety and efficacy.

\section{Materials and methods}

We reviewed the PubMed English literature of the last 10 years about ESD and Z-POEM for ZD treatment. The following keywords were used: Zenker diverticulum, stapling diverticulostomy, endoscopic stapling diverticulostomy, Z-POEM, flexible endoscopy. Clinical review, prospective and retrospective studies were included. Comparative studies between different techniques were considered only if stratified and differentiated data were reported. Studies with recurrent diverticula treatment reported with naïve together, were excluded.

Patient and diverticulum characteristics, surgical procedure techniques, technical success rate, clinical success rate, recurrence rate and complications were analysed.

\section{Results}

The literature search and selection produced 20 papers. Eleven articles about ESD for a total of 1,490 patients and 9 articles about Z-POEM for a total of 96 patients.

Two studies out of 20 were prospective $(6,7), 11$ were retrospective (8-18), 6 case reports (19-24) and 1 clinical review (25).

Of the retrospective studies, 6 were comparative studies between ESD and other treatment techniques, such as the flexible endoscopic treatment (9), $\mathrm{CO}_{2}$ laser (11-13), open surgery $(12,14,15)$ or a new technique called MTORD (transoral resection of diverticulum) (15). About Z-POEM we reviewed 6 case reports, 1 retrospective study and 2 comparative retrospective studies where the Z-POEM's outcomes were compared to the classic endoscopic septotomy $(17,18)$. All the studies about ESD are summarized in the Table 1 and those about Z-POEM in Table 2.

\section{Discussion}

\section{Surgical procedures}

\section{ESD}

The procedure is performed under general anaesthesia with oro-tracheal intubation. The patient is positioned in supine position with the head extended. A rigid Dohlman or Weerda diverticuloscope is transorally inserted with a blade inside the diverticulum and the other one inside the esophagus, in order to expose the septum. After that, the diverticuloscope is fixed over the chest by a lever arm. Diverticuloscope positioning is the most difficult phase of the procedure because kyphosis, retrognathia, large tongue or dental abnormalities can make it impossible. Once the septum is exposed, a $30 \mathrm{~mm}$ linear stapler is inserted and fired to divide the septum. This step can be repeated until a complete section is obtained. The main problem is that the tip of the cartridges is not active in dividing and suturing, thus always leaves the distal part of the septum untreated for some millimeters. To overcome this drawback some authors modify by themselves the cartridges, cutting away the inactive tip and applying 2 endostitches to the septum in order to countertract it and pushing deeper the stapler $(6,7)$.

\section{Z-POEM}

$\mathrm{Z}$-POEM is performed under general anaesthesia with 


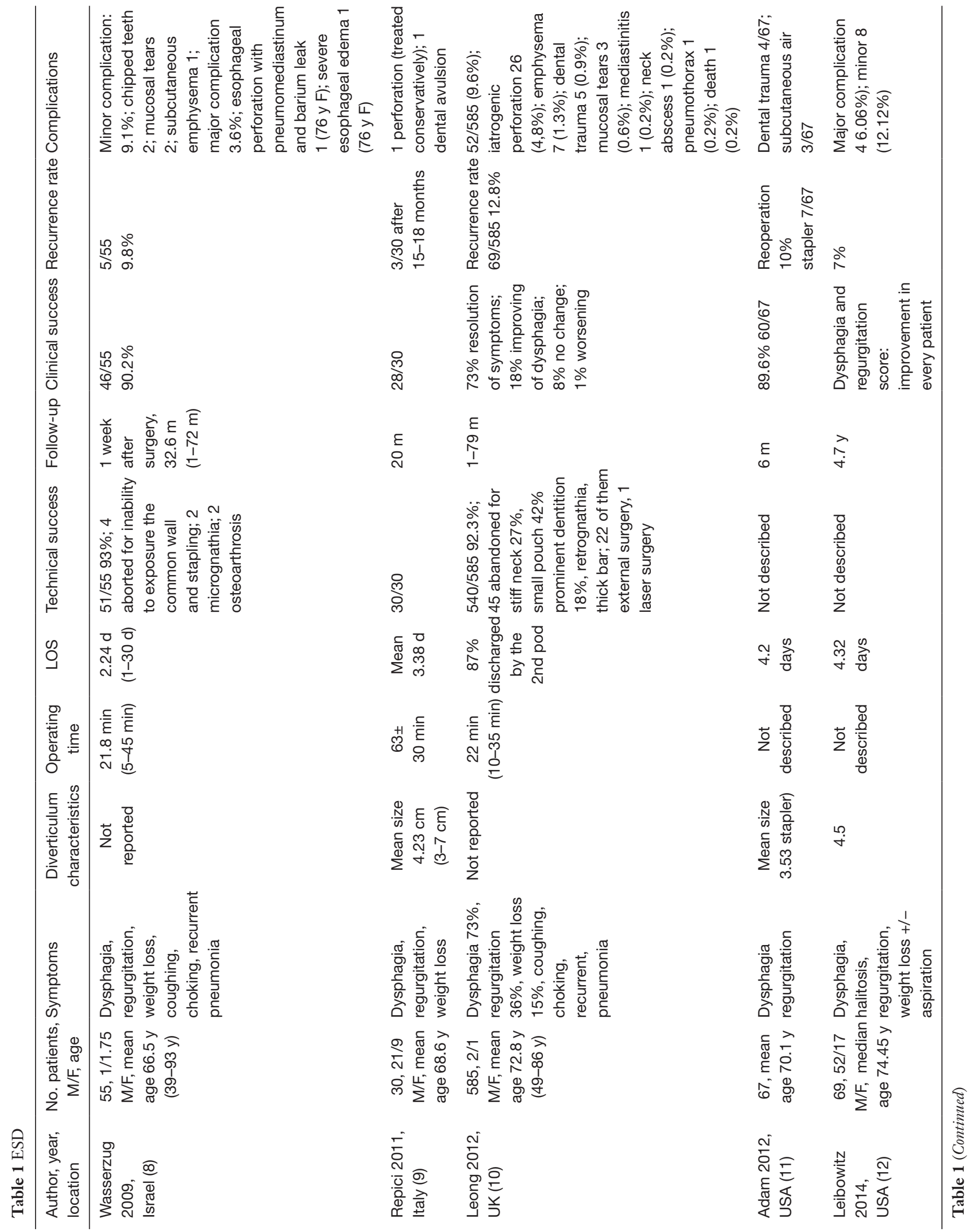




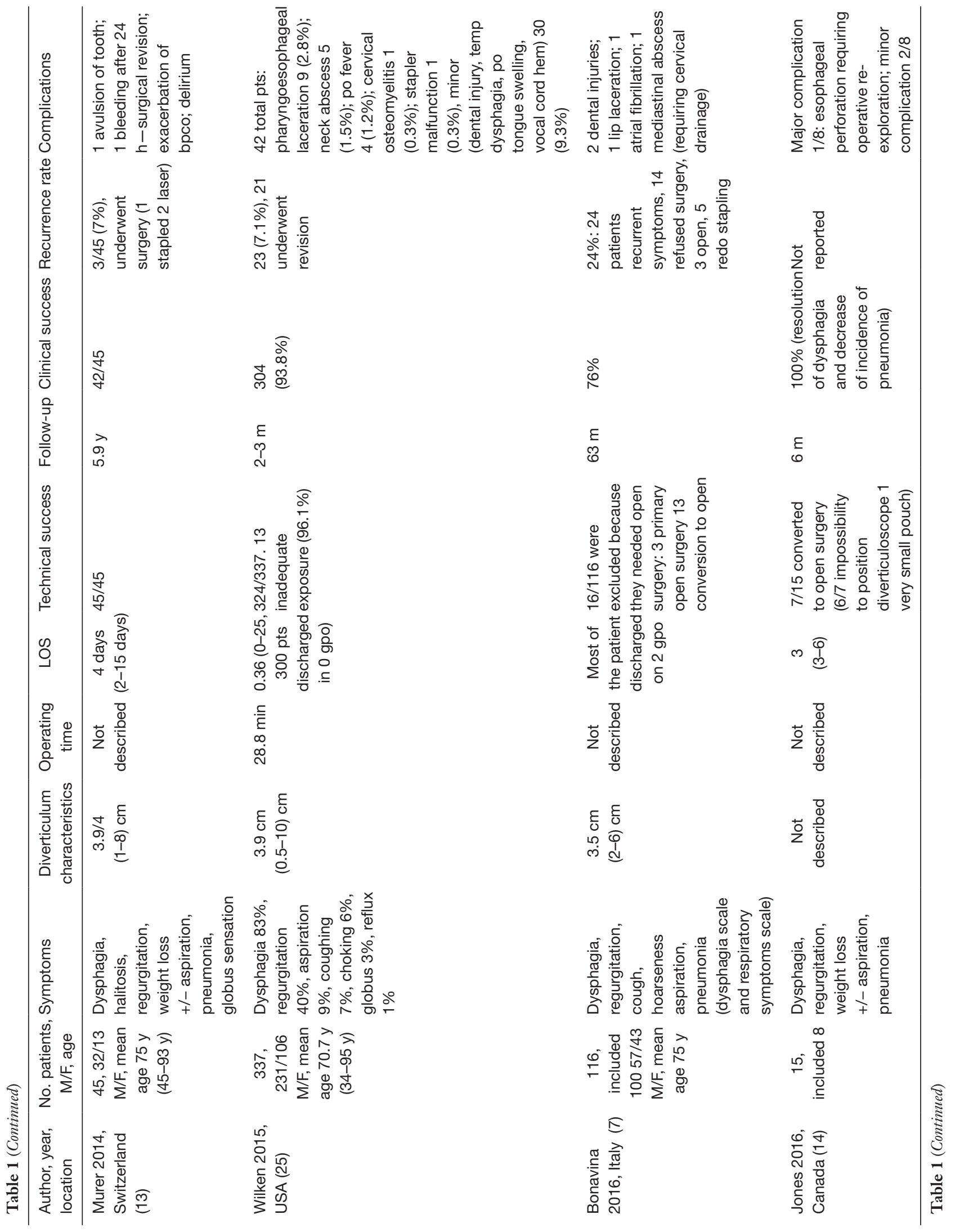



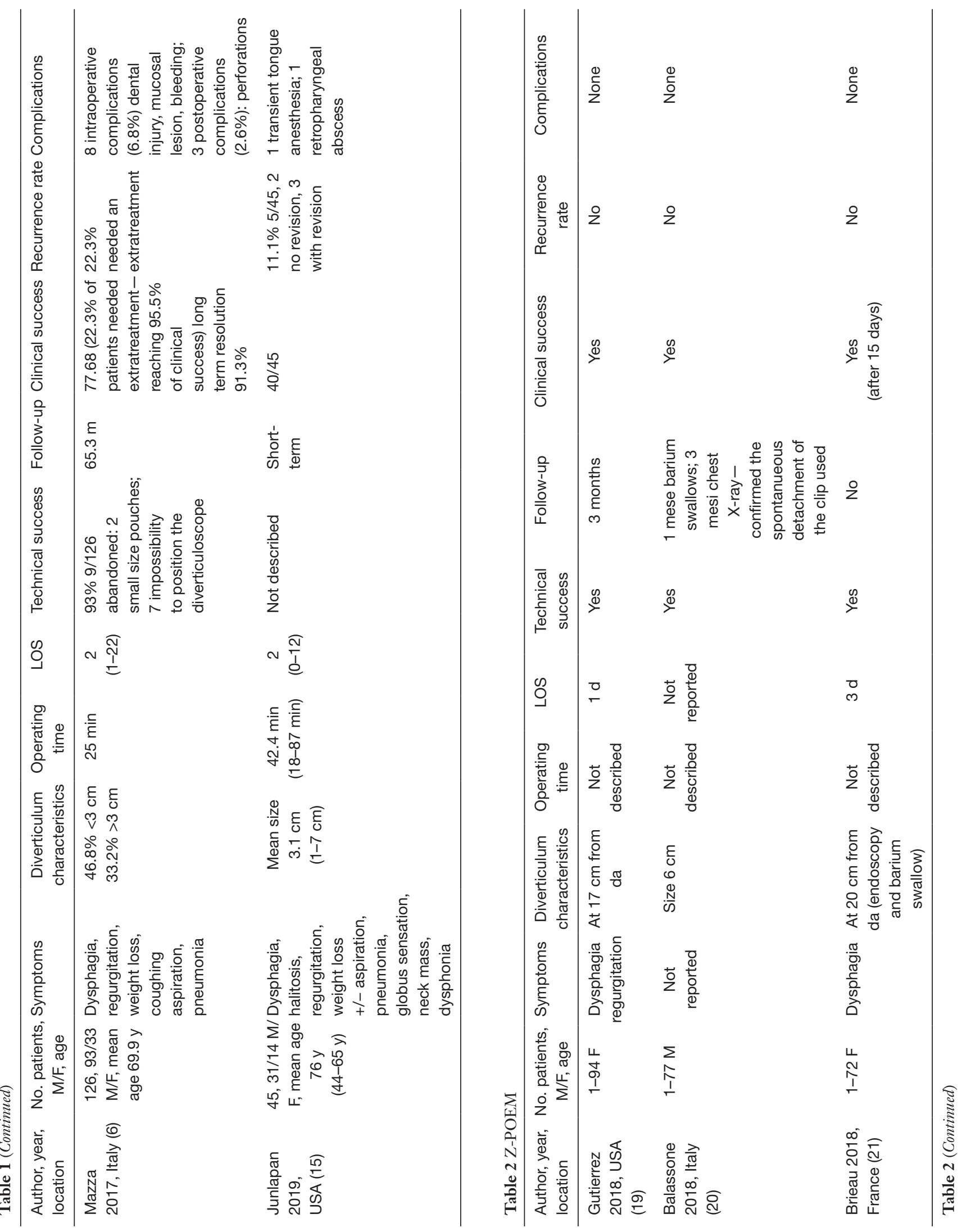


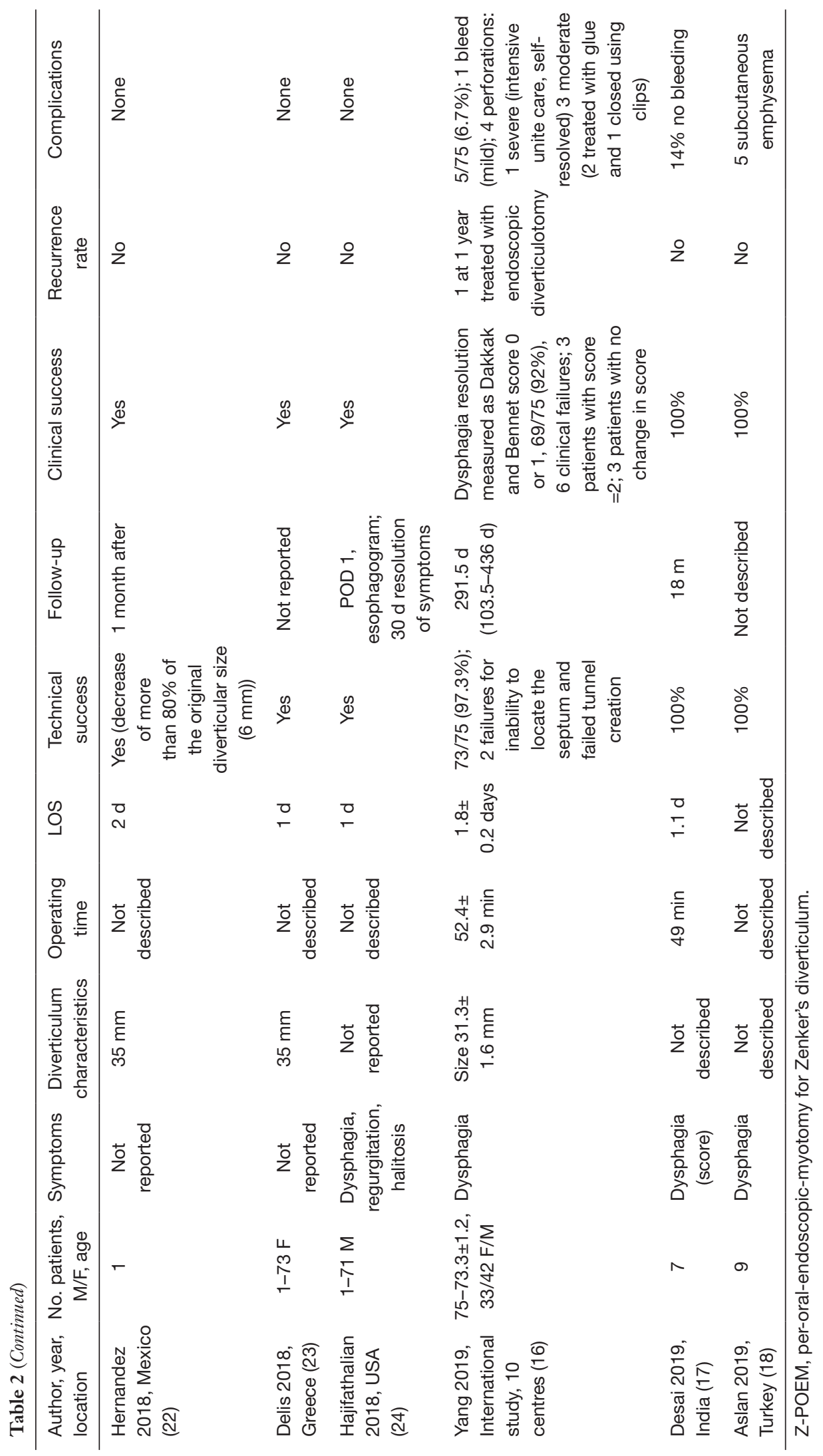


oro-tracheal intubation. A standard gastroscope, with or without a clear cup, is used for the procedure. After the identification of the ZD, a mucosal bleb is created $1-2 \mathrm{~cm}$ proximal to the diverticular septum, by injecting a solution of indigo carmine, saline solution and epinephrine. A mucosal incision of $1-1.5 \mathrm{~cm}$ is performed to create the submucosal tunnel entry. The incision is done using a triangle tip-knife or a hook-knife, according to the physician preferences, and the submucosal tunnel is created using the same device and spray coagulation. Once the septum is reached, tunnelling is extended on both sides, and when it is completely exposed, the septotomy is performed by dividing the muscular fibres of the UES and of the first centimetres of the esophagus leaving intact the mucosal layer. The endoscope is then retracted and the mucosal entry closed by endoscopic clips application (26).

This procedure provides the treatment of the UES only and leaves the diverticular sac intact, while the previous one provides a complete section of the UES and treats the diverticulum creating a common room with the cervical esophagus.

\section{Patient characteristics}

The mean age of patients submitted to ESD was 72.4 years $(39-95$ years) $(8,25)$ while of those treated with Z-POEM was 76.7 years (71-94 years) $(19,24)$. These data confirm that the ZD is basically a disorder of male elderly population and that the age is not a discriminant in decision about which technique apply. The mean diverticular dimension was $3.82 \mathrm{~cm}(0.5-10 \mathrm{~cm})(25)$ among those treated with ESD and $3.14 \mathrm{~cm}(2-6 \mathrm{~cm})(16)$ among those submitted to Z-POEM. The wide variability in diverticular sac size suggests that dimension too is not a discriminant in choosing the treatment.

\section{Operating time}

The mean operating time of ESD was $26.6 \mathrm{~min}(5-87 \mathrm{~min})$ $(8,15)$ while was $52.1 \mathrm{~min}$ for Z-POEM procedure $(16,17)$. Z-POEM takes longer time than the ESD but for different reasons. ESD has a higher variability mainly due to the difficulty of diverticuloscope positioning, but the stapling time is quite fast. Besides, the Z-POEM procedure takes almost always longer time with less variability (52.4 $\mathrm{min} \pm$ $2.9 \mathrm{~min}$ ), probably because the myotomy technique requires a careful muscular fibres section (16).

\section{Length of stay}

The mean length of hospital stay for ESD was 1.78 days (0-30 days) $(8,25)$, while for Z-POEM was 1.75 days (128 days) (16). Both procedures have similar hospital stay in most authors. This is probably due to the fact that both are endoscopic procedure with the same clinical impact and clinical management, so most of the patient are discharged within the second postoperative day. Anyway the discrepancy between the number of patients between the two groups doesn't allow conclusive evaluations.

\section{Technical success}

Technical success was defined by the ability to perform the procedure. Technical failure of ESD are mostly due to problems in diverticuloscope positioning and consequently the exposure of the septum. Neck stiffness, osteoarthrosis, micrognathia, prominent dentition, retrognathia are the most common reasons.

Wasserzug et al. reported 4 failures (7.3\%) out of 55 patients for inability to adequately expose the septum: 2 for micrognathia and 2 for osteoarthrosis (8). Similarly Leong et al. reported 45 technical failures out of $585(7.7 \%)$ for neck stiffness (27\%), prominent dentition (18\%); 22 of them underwent open surgery and one laser surgery (10).

Bonavina et al. showed that the degree of mouth opening was significantly associated to the conversion rate. They used different scores, such as Mallampati, Cormack scores, incisor distance and thyromental distance, showing that patients undergoing uneventuful transoral approach had a larger mouth opening compared to individuals requiring conversion to open surgery (7).

A small size of the diverticular sac is another important reason of technical failure of ESD, being responsible from $22.2 \%$ to $42 \%$ of all failed cases $(6,10)$. In fact, if a diverticulum is less than $2 \mathrm{~cm}$ large, it's not possible to have a complete section of the UES fibres thus not abolishing the "primum movens" of the disease.

The Z-POEM doesn't require the positioning of a rigid diverticuloscope, so neck or oral cavity abnormalities are not a problem for the procedure. The only two cases reported in literature, in which the procedure was not possible, were for the inability to locate the septum and failure to create the submucosal tunnel (16).

Z-POEM seems to have a higher technical success rate of $97.9 \%$. This could be explained by a lower dependency of the technique from patient's anatomy and devices 
positioning, even if the pure section of the septum appears to be more difficult than ESD.

\section{Clinical outcomes}

The clinical success was defined as the resolution of the symptoms after surgery or at least a significant improvement of them (1).

In literature ESD is reported to have a high clinical success rate. Leong et al. evidenced a success rate of $91 \%$, reporting $73 \%$ of patients with a complete resolution of dysphagia and $18 \%$ with an improvement of dysphagia, $8 \%$ with no change and only $1 \%$ had a worsening (10).

Similar results are described by the most of authors $(8-13,15,25)$. The only two prospective studies present in literature showed good clinical results in $77.68 \%$ and $76 \%$ respectively $(6,7)$. In particular $22.3 \%$ of patients needed an extra treatment to have a complete resolution of the symptoms, thus reaching a success rate of $95.5 \%$ (6). Rosati (6) argued that an incomplete stapling of the septum was the main cause of failure and that an extension of the section was needed.

Bonavina et al., besides, showed that the clinical success is significantly associated with age, diverticulum size and endostich use. In particular the success rate was higher in patients older than 70, with a ZD size larger than $3 \mathrm{~cm}$ and when the traction sutures was used (7).

Clinical results of Z-POEM in symptoms solutions seem to be similarly good. Yang et al. by the use of Dakkak and Bennet score (16) reported in their paper a success rate of $92 \%: 78.7 \%$ with a dysphagia score $0,17.3 \%$ with a dysphagia score 1 ; the $8 \%$ with no change in dysphagia score or with a score 2 was classified as clinical failure. All the various clinical cases published reported a good result of the procedure (19-24).

Even in this case the wide difference between the dimensions of the two populations doesn't allow to take definitive conclusions. Probably the efficacy of the myotomy of the Z-POEM is high, but concerns still remains about the effect of the persistence of the diverticular pouch, particularly on symptoms such as regurgitation and aspiration.

\section{Persistence and recurrence of symptoms}

Recurrence was defined as the reappearance of symptoms during the follow up of patients classified initially as a clinical success, while the persistence was defined as the worsening or no change in symptoms after the surgery (1).

Wilken et al. described a success rate of $93.8 \%$ with ESD but, 20 patients (6.2\%) failed to improve after the treatment and were considered as persistence of symptoms (19 patients out of 337 had no change and one patient referred a worsening) (25). Similarly Leong described 9\% of persistence: $8 \%$ no change and $1 \%$ of worsening (10).

Furthermore, Rosati and other authors evidenced how the use of the endostitches for countertraction improved the success rate after a single treatment session, reducing the need for retreatment $(6,27,28)$. This is probably due to the traction on tissue that can improve the stapling inducing a really complete section of the septum.

The recurrence rate after ESD is reported to be around $10 \%$ by different authors $(8,9,11)$; Leibowitz et al. describe a $7 \%$ of recurrence rate (12), similarly to Wilken et al, with a $7.1 \%(25)$. Higher recurrence rate is reported by Leong et al. with $12.8 \%$ (10). Others evidenced how the recurrence rate is significantly higher only in patients with a diverticulum $<3 \mathrm{~cm}(7)$.

Among 96 patients treated with Z-POEM and published in literature in only 1 case an extra endoscopic diverticulotomy was required for recurrent symptoms (16), apparently having a recurrence rate of $1.04 \%$. Again, the low numbers of published procedures of Z-POEM makes this value poorly reliable.

\section{Complications}

Endoscopic rigid or flexible techniques were developed just in order to reduce the incidence and the entity of complications induced by the open surgical approach and nowadays the most common complications of endoscopic approaches are the minor ones.

Dental avulsion or chipped teeth and lips laceration are the most common intraoperative complications and are described only in the ESD technique. The main reason of these complications is the difficult insertion of the rigid diverticuloscope itself through the oral cavity. They globally account for $4.5 \%$ of cases (6).

Among major complications the most frequent are bleeding and perforation. Perforations include mucosal tears and lacerations that could theoretically lead to a mediastinal abscess, neck and retropharyngeal abscess, emphysema, pneumothorax requiring surgery and $\backslash$ or drainage.

Four-point eight percent of iatrogenic perforations were 
described after ESD, with 1 case $(0.2 \%)$ of mediastinitis, 1 pneumothorax and 1 neck abscess. 1 death only was described for mediastinitis after a perforation unsuccessfully treated with a pectoralis major flap repair (10).

Other authors reported a perforation rate ranging from 0 to $2.8 \%(6,25,28)$. All cases were conservatively treated.

The bleeding is quite rare too, accounting for about $2 \%$ of cases; this complication can occur intraoperatively or postoperatively but is often self-limiting and easily controlled with electric coagulation (6).

The Z-POEM too is burdened by a low complication rate. Yang et al. reported 1 mild bleeding and 4 perforations that were treated with glue or clips; 1 of them required an intensive unit care admission, but self-resolved anyway (16). Aslan $e t a l$. reported 5 patients out of 9 with self-limiting subcutaneous emphysema and other authors had no morbidity (18).

Both procedures seem to be safe and burdened by a low complication rate. Anyway the risk of severe complications, such as mediastinitis, must always be kept in mind, because mortality it's not null. The low number of Z-POEM procedures published should still induce a careful stance because the risks could be underestimated.

\section{Conclusions}

The endoscopic treatment of ZD, irrespectively of ESD or Z-POEM, have been proved safe, because the incidence and severity of complication is low, even if sometimes severe adverse events can occur.

Both the techniques are feasible in most of cases, even if the ESD is burdened by the difficult positioning of the rigid diverticuloscope that can lead to a lower technical success rate and that is responsible for the majority of minor complications.

The ESD appears to be a faster than Z-POEM procedure in most cases, even if sometimes, when a difficult diverticuloscope positioning is present, becomes a timeconsuming procedure.

The effectiveness of both procedures is high, with a symptoms solution rate of about $90 \%$. Nevertheless, some technical tips, such as the endostitches use, should be adopted for ESD to improve his clinical success rate. Moreover, a better definition of clinical results is needed particularly for Z-POEM patients. In fact, concerns are present about the remaining of the diverticular pouch intact, that could lead to a persistence of some symptoms as regurgitation and aspiration.

Nowadays ESD appears to be a well-consolidated and evaluated technique, being applied since 1993, on the contrary the Z-POEM, even if seductive and fashionable, needs a thorough evaluation of its results before obtaining a wide diffusion and should be applied only by experienced endoscopists.

\section{Acknowledgments}

Funding: None.

\section{Footnote}

Provenance and Peer Review: This article was commissioned by the Guest Editor (Ghulam Abbas) for the series "Minimally Invasive Esophageal Surgery" published in Shangbai Chest. The article has undergone external peer review.

Conflicts of Interest: All authors have completed the ICMJE uniform disclosure form (available at http://dx.doi. org/10.21037/shc.2020.01.04). The series "Minimally Invasive Esophageal Surgery" was commissioned by the editorial office without any funding or sponsorship. The authors have no other conflicts of interest to declare.

Ethical Statement: The authors are accountable for all aspects of the work in ensuring that questions related to the accuracy or integrity of any part of the work are appropriately investigated and resolved.

Open Access Statement: This is an Open Access article distributed in accordance with the Creative Commons Attribution-NonCommercial-NoDerivs 4.0 International License (CC BY-NC-ND 4.0), which permits the noncommercial replication and distribution of the article with the strict proviso that no changes or edits are made and the original work is properly cited (including links to both the formal publication through the relevant DOI and the license). See: https://creativecommons.org/licenses/by-nc-nd/4.0/.

\section{References}

1. Deepanshu J, Abhinav S, Manan S, et al. Efficacy and safety of flexible endoscopic management of Zenker's diverticulum. J Clin Gastroenterol 2018;52:369-85. 
2. Wheeler WJ. Pharyngocele and dilation of pharynx. Dublin J Med Sci 1886;82:349-57.

3. Mosher HP. Webs and pouches of the esophagus: their diagnosis and treatment. Surg Gynecol Obstet 1917;25:175-87.

4. Collard JM, Otte JB, Kestens PJ. Endoscopic stapling technique of esophagodiverticulostomy for Zenker's diverticulum. Ann Thorac Surg 1993;56:573-6.

5. Li QL, Chen WF, Zhang XC, et al. Submucosal tunnelling endoscopic septum division: a novel technique for treating Zenker's diverticulum. Gastroenterology 2016;151:1071-4.

6. Mazza M, Bergamini AN, Parise P, et al. Treatment of Zenker's diverticulum with endoscopic stapled esophagodiverticulostomy: Analysis of long-term outcome. Surg Laparosc Endosc Percutan Tech 2017;27:445-8.

7. Bonavina L, Aiolfi A, Scolari F, et al. Long-term outcome and quality of life after transoral stapling for Zenker diverticulum. World J Gastroenterol 2015;21:1167-72.

8. Wasserzug O, Zikk D, Raziel A, et al. Endoscopically stapled diverticulostomy for Zenker's diverticulum: results of a multidisciplinary team approach. Surg Endosc 2010;24:637-41.

9. Repici A, Pagano N, Fumagalli U, et al. Transoral treatment of Zenker diverticulum: flexible endoscopy versus endoscopic stapling. A retrospective comparison of outcomes. Dis Esophagus 2011;24:235-9.

10. Leong SC, Wilkie MD, Webb CJ. Endoscopic stapling of Zenker's diverticulum: establishing national baselines for auditing clinical outcomes in the United Kingdom. Eur Arch Otorhinolaryngol 2012;269:1877-84.

11. Adam SI, Paskhover B, Sesaki CT. Laser Versus Stapler: Outcomes in endoscopic repair of Zemler Diverticulum. Laryngoscope 2012;122:1961-6.

12. Leibowitz JM, Fundakowski CE, Abouyared M, et al. Surgical Techniques for Zenker's Diverticulum: A Comparative Analysis. Otolaryngol Head Neck Surg 2014;151:52-8.

13. Murer K, Soyka MB, Broglie MA, et al. Zenker's diverticulum: outcome of endoscopic surgery is dependent on the intraoperative exposure. Eur Arch Otorhinolaryngol 2015;272:167-73.

14. Jones D, Aloraini A, Gowing S, et al. Evolving management of Zenker's diverticulum in the endoscopic era: a north American experience. World J Surg 2016;40:1390-6.

15. Junlapan A, Abu-Ghanem S, Sung CK, et al. Outcomes in modified transoral resection of diverticula for Zenker's diverticulum. Eur Arch Otorhinolaryngol
2019;276:1423-9.

16. Yang J, Novak S, Ujiki M, et al. An international study on the use of peroral endoscopic myotomy in the management of Zenker's diverticulum. Gastrointest Endosc 2020;91:163-8.

17. Deasai PN, Kabrawala MV. Submucosal tunnelling endoscopic septum division for Zenker's Diverticulum (Z-POEM): a new emerging technique compared to conventional flexible endoscopic septotomy. Gastrointest Endosc 2019;89:AB450.

18. Aslan F, Yilmaz O, Sengun B, et al. A new technique in treatment of Zenker Diverticulum: Submucosal tunnelling endoscopic septum division (Z.POEM) versus classic endoscopic septotomy techniques. Gastrointest Endosc 2019;89:AB629.

19. Brewer Gutierrez OI, Moran R, Yang J, et al. Successful single-session cricopharyngeal and Zenker's diverticulum peroral endoscopic myotomy. Endoscopy 2018;50:E220-1.

20. Balassone V, Pizzicannella M, Biasutto D, et al. Submucosal per-oral endoscopic myotomy for a large Zenker's diverticulum with use of a hydrodissector knife and an over-the-scope clip closure. VideoGIE 2018;3:373-4.

21. Brieau B, Leblanc S, Boardcahar B, et al. Submucosal tunnelling endoscopic septum division for Zenker's diverticulum: a reproducible procedure for endoscopists who perform peroral endoscopic myotomy. Endoscopy 2017;49:613-4.

22. Hernández Mondragón OV, Solórzano Pineda MO, Blancas Valencia JM. Zenker's diverticulum: Submucosal tunneling endoscopic septum division (Z-POEM). Dig Endosc 2018;30:124.

23. Delis K, Robotis J, Sachitzi E, et al. Submucosal tunneling endoscopic septum division of a Zenker's diverticulum. Ann Gastroenterol 2018;31:634.

24. Hajifathalian K, Dawod Q, Saumoy M, et al. Submucosal tunneling endoscopic septum division for treatment of Zenker's diverticulum. Endoscopy 2018;50:E340-1.

25. Wilken R, Whited C, Scher RL. Endoscopic staple diverticulostomy for Zenker's diverticulum: review of experience in 337 cases. Ann Otol Rhinol Laryngol 2015;124:21-9.

26. Brewer Gutierrez OI, Ichkhanian Y, Spadaccini M, et al. Zenker's Diverticulum Per-Oral Endoscopic Myotomy Techniques: Changing Paradigms. Gastroenterology 2019;156:2134-5.

27. Bonavina L, Rottoli M, Bona D, et al. Transoral stapling 
for Zenker diverticulum: effect of the traction sutureassisted technique on long-term outcomes. Surgical Endoscopy 2012;26:2856-61.

28. Provenzano L, Salvador R, Cutrone C, et al. Traction

doi: $10.21037 /$ shc. 2020.01 .04

Cite this article as: Cerchione R, Parise P, Olivari G, Cossu A, Barbieri L, Palucci M, Cinelli L, Puccetti F, Rosati R. Endoscopic management of Zenker's diverticulum: stapling vs. Z-POEM. Shanghai Chest 2020;4:43. on the septum during transoral septotomy for Zenker diverticulum improves the final outcome. Laryngoscope 2020;130:637-40. 